\title{
Sur l'aspect de la planète Saturne \\ et spécialement sur une tache blanche et brillante observée sur son anneau.
}

\author{
(Schreiben von Dr. F. Terby in Loewen an den Herausgeber.)
}

J'ai eu l'honneur de vous adresser aujourd'hui une courte dépêche pour vous informer de la présence d'une région claire et blanche sur l'anneau de Saturne, contre l'ombre que projette le globe. Mon but etait de vous mettre à mêne de vérifier ce fait intéressant aujourd'hui dejà si le temps est favorable. Je vous écris la présente lettre pour vous donner de plus amples détails. Le petit dessin ci-joint me fera comprendre.

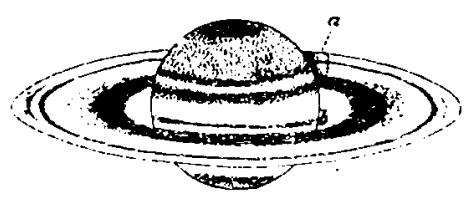

L.e 6 mars, ḋ $9^{\mathrm{h}} \mathbf{1}^{\mathrm{m}}$, je remarquai que la région $a$, pointillée dans ce dessin, s'étendant à la fois sur les anneaux $A$ et $B$, tranchait sur tout le reste des anneaux par son vif éclat et sa lumière blanche; je ne puis la comparer qu'à ces taches blanches et brillantes qui s'observent souvent sur Jupiter et dont on s'est beauconp occupé depuis quelques années. Je croyais tenir l'occasion de vérifier le mouvement de rotation des anneaux par le déplacement de cette tache, mais à $9^{\mathrm{h}} 49^{\mathrm{m}}$ aucun changement ne se manifestait encore et la région $a$ me semblait même avoir perdu de son éclat; il est vrai que le ciel devenait de plus en plus nébuleux et qu'à ${ }_{1} 0^{h} 24^{\mathrm{m}}$ l'observation ne pouvait plus se poursuivre.

Le 12 mars, à $9^{\mathrm{h}} 20^{\mathrm{m}}$, la tache était de nouveaus visible, un ciel couvert ayant enpêché les observations les jours précédents; cette fois sa blancheur et son éclat etaient encore frappants et la division Cassinienne, qui la traversait, semblait $y$ gagner en noirceur et en visibilité par l'effet du contraste. Ces observations ont été faites surtout avec les grossissements de 250 et de 280 du huit pouces de Grubb, mais 150 montrait aussi très bien cette blancheur. Je poursuivis l'observation jusqu'à $10^{\mathrm{h}} 35^{\mathrm{m}}$, moment où le ciel se couvrit complètement et je ne constatai aucun déplacement dans la tache; j'annotai seulement que son éclat semblait avoir diminué; mais cette fois encore le ciel devenait nébuleux.

Pour continuer ce qui concerne les anneaux je dirai que la bande d'Encke se remarquait mieux sur l'anse occidentale que sur l'anse orientale; mais le I 2, pourtant, elle était aussi fort difficile sur l'anse occidentale. Je suis frappé aussi de la visibilité excessive de la division Cassinienne devant la planète, malgré le rétrécissement progressif des anneaux; elle me semble renforcée cette année dans cette région; le bord externe de l'anneau, par contre, se voit difficilement devant la planete. L'anneau $B$ est visiblement, quoique légèrement, ombré du côté interne. Quant à l'anneau obscur il est d'une évidence et d'une noirceur frappantes devant la planète; c'est, après l'ombre du globe et la division de Cassini, la région la plus noire de tout le système de Saturne. La bande de Struve est apparue généralement comme je la figure et surtout au commencement des observations de ces deux soirées; à droite c'était un croissant noir, large, à l'intérieur duquel semblait flotter l'anneau $C$ complètement séparé de $B$; à gauche c'était une strie noire allant du point où l'anneau émerge de derrière le globe jusqu'à la pointe occidentale de la lunule. Mais, fort peu de temps après, ces aspects semblaient modifiés et portaient à admettre un mouvement excessivement rapide de l'anneau. La délicatesse de ces observations empêchera probablement longtemps encore d'éclaircir le nyystère.

L'ombre du globe sur les anneaux est peut-être légèrement concave mais je n'ai su y voir aucune inégalité sur la division Cassinienne, comparable à ce que j'ai vu en $1886-1887$ et $1887-1888$.

La disposition des bandes sur le globe n'a point varié depuis 1886 ; il faut dire seulement que, le bord nord du disque se découvrant, on commence à y voir une ombre séparée du bord externe de l'anneau, peut-être une bande septentrionale, ou le bord de la calotte polaire de cet hémisphère.

Je crois devoir appeler spécialement l'attention sur la bande fine, grise, $b$; elle longe la projection de l'anneau obscur sur la planète et je l'observe depuis 1886, c'est à dire depuis que je me sers de mon huit pouces. Cette bande délicate, qui ne figure pas encore dans beaucoup de dessins autérieurs, pouvait, selon sa position, devenir de plus en plus difficile à observer par le fait du rétrécissement de l'anneau; en 1887-1888 c'était déjà un objet assez difficile; actuellement elle se voit moins aisément qu'en I 886 - 1887 surtout, mais demeure un objet très évident.

Si l'un me permet de clore cet exposé de faits positifs par une conjecture assez plausible, me semble-t-il, je me hasarderai à dire que l'avenir nous apprendra peut-être si cette bande singulière appartient reellement au globe de la planète ou n'est pas la projection d'un second anneau obscur très étroit qui nous échappe encore dans les lunules conme l'anneau obscur connu s'y est caché pendant si longtemps. Nul ne saurait prévoir les découvertes que la photographia de l'avenir nous réserve dans le système saturnien, pas plus que nul n'eût osé s'attendre aux admirables résultats que la photographie actuelle donne sur la constitution physique des nébuleuses. 